\title{
AN EXTENDED POSSIBILITY-BASED FUZZY NESTED RELATIONAL DATABASE MODEL AND ALGEBRA
}

\author{
Z. M. Ma and F. Mili \\ Department of Computer Science and Engineering, Oakland University, Rochester, MI 48309
}

\section{INTRODUCTION}

In order to enhance the expressive power of relational database model, non-first normal $\left(\mathrm{NF}^{2}\right.$ or nested) relational database model has been introduced, where attribute values may be atomic or set-valued and even relations themselves [1], on one hand. On the other hand, Fuzzy values have been employed to model information imprecision in databases since Zadeh [4] proposed the theory of fuzzy sets. A NF ${ }^{2}$ relational database model with fuzzy information accommodates imprecision and complexity of the real world objects simultaneously. Based on similarity relation, a fuzzy $\mathrm{NF}^{2}$ relational database model is introduced in [3] and two restructuring operations: Merge and Unmerge are emphatically given. In this paper, an extended possibility-based fuzzy $\mathrm{NF}^{2}$ relational database model is introduced, where the fuzziness of data comes from possibility distributions over universes of discourse and as resemblance relation [2]. We give database model and define fuzzy nested algebra.

\section{DATABASE MODEL}

A fuzzy $\mathrm{NF}^{2}$ relational schema is a set of attributes (A1: D1, A2: D2,.., An: Dn), where attribute domain $\mathrm{Di}(1 \leq \mathrm{i} \leq \mathrm{n})$ can be one of the following: (1) The set of atomic values, denoted dom; (2) The set of null values, denoted ndom, where null values may be unk, inap, nin, and onul; (3) The set of fuzzy subset, denoted fdom; (4) The power set of the set in (1), denoted sdom; (5) 
The set of relation values. The corresponding attribute value, say ai, is a tuple of the form <ai1, ai2, ..., aim> which is an element of Di1 $\times$ Di $2 \times \ldots \times$ Dim $(m>1$ and $1 \leq \mathrm{i} \leq \mathrm{n})$, where each $\mathrm{Dij}(1 \leq \mathrm{j} \leq \mathrm{m})$ may be a domain in (1), (2), (3), and (4) and even the set of relation values. Formally, the attribute domain of $A_{i}(1 \leq i \leq n)$ is represented as follows.

$$
\tau_{\mathrm{i}}=\text { dom } \mid \text { ndom } \mid \text { fdom } \mid \text { sdom } \mid<\mathrm{B}_{1}: \tau_{\mathrm{i} 1}, \mathrm{~B}_{2}: \tau_{\mathrm{i} 2}, \ldots, \mathrm{B}_{\mathrm{m}}: \tau_{\mathrm{im}}>
$$
where $\mathrm{B} 1, \mathrm{~B} 2, \ldots, \mathrm{Bm}$ are attributes.

A relation $r$ over fuzzy $\mathrm{NF}^{2}$ schema $\left(\mathrm{A}_{1}: \tau_{1}, \mathrm{~A}_{2}: \tau_{2}, \ldots, \mathrm{A}_{\mathrm{n}}: \tau_{\mathrm{n}}\right)$ is a subset of Cartesian product $\tau_{1} \times \tau_{2} \times \ldots \times \tau_{\mathrm{n}}$. A tuple in $r$ with the form of $<\mathrm{a} 1, \mathrm{a} 2$, $\ldots$, an $>$ consists of $n$ components. Each component ai $(1 \leq \mathrm{i} \leq \mathrm{n})$ may be an atomic value, null value, set value, fuzzy value, or another tuple.

The semantic relationships between two fuzzy data include equivalence, inclusion, intersection, and irrelevancy, which can be assessed with semantic inclusion degree proposed in [2].

Let $\pi_{\mathrm{A}}$ and $\pi_{\mathrm{B}}$ be two fuzzy data on $U=\left\{\mathrm{u}_{1}, \mathrm{u}_{2}, \ldots, \mathrm{u}_{\mathrm{n}}\right\}$. Let Res be a resemblance relation on $U$ and $\alpha(0 \leq \alpha \leq 1)$ be a threshold corresponding to Res. The semantic inclusion degree of $\pi_{\mathrm{A}}$ and $\pi_{\mathrm{B}} \operatorname{SID}_{\alpha}\left(\pi_{\mathrm{A}}, \pi_{\mathrm{B}}\right)$ based on $\alpha$, which means $\pi_{\mathrm{A}}$ semantically includes $\pi_{\mathrm{B}}$, is defined as

$$
\operatorname{SID}_{\alpha}\left(\pi_{\mathrm{A}}, \pi_{\mathrm{B}}\right)=\sum_{i=1}^{\mathrm{n}} \min _{\mathrm{u}_{i}, u_{\mathrm{j}} \in \mathrm{D}}\left(\pi_{\mathrm{B}}\left(\mathrm{u}_{\mathrm{i}}\right), \pi_{\mathrm{A}}\left(\mathrm{u}_{\mathrm{i}}\right)\right) / \sum_{i=1}^{\mathrm{n}} \pi_{\mathrm{B}}\left(\mathrm{u}_{\mathrm{i}}\right)
$$

For a given threshold $\beta$, we have (1) $\pi_{A}$ includes $\pi_{B}$ iff $\operatorname{SID}_{\alpha}\left(\pi_{A}, \pi_{B}\right) \geq \beta$, (2) $\pi_{\mathrm{A}}$ and $\pi_{\mathrm{B}}$ are equivalent to each other iff $\operatorname{SID}_{\alpha}\left(\pi_{\mathrm{A}}, \pi_{\mathrm{B}}\right) \geq \beta$ and $\operatorname{SID}_{\alpha}\left(\pi_{\mathrm{B}}\right.$, $\left.\pi_{\mathrm{A}}\right) \geq \beta$, and (3) $\pi_{\mathrm{A}}$ and $\pi_{\mathrm{B}}$ are irrelevant to each other iff $\operatorname{SID}_{\alpha}\left(\pi_{\mathrm{A}}, \pi_{\mathrm{B}}\right)<\beta$ and $\operatorname{SID}_{\alpha}\left(\pi_{B}, \pi_{A}\right)<\beta$. The notion of equivalence degree for two fuzzy data is hereby given as follows.

The semantic equivalent degree of $\pi_{\mathrm{A}}$ and $\pi_{\mathrm{B}} \mathrm{SE}_{\alpha}\left(\pi_{\mathrm{A}}, \pi_{\mathrm{B}}\right)$, denoting the degree that $\pi_{\mathrm{A}}$ and $\pi_{\mathrm{B}}$ are equivalent to each other, is hereby defined.

$$
\operatorname{SE}_{\alpha}\left(\pi_{\mathrm{A}}, \pi_{\mathrm{B}}\right)=\min \left(\operatorname{SID}_{\alpha}\left(\pi_{\mathrm{A}}, \pi_{\mathrm{B}}\right), \operatorname{SID}_{\alpha}\left(\pi_{\mathrm{B}}, \pi_{\mathrm{A}}\right)\right)
$$

Two fuzzy data $\pi_{\mathrm{A}}$ and $\pi_{\mathrm{B}}$ are considered $\alpha-\beta$-redundant iff $\mathrm{SE}_{\alpha}\left(\pi_{\mathrm{A}}, \pi_{\mathrm{B}}\right)$ $\geq \beta$. The equivalent degree of two crisp data (atomic or set-valued) is 1.0 if they are equal to each other. The elimination of duplicate could be achieved by merging $\pi_{\mathrm{A}}$ and $\pi_{\mathrm{B}}$ and producing a new fuzzy data $\pi_{\mathrm{C}}$. We define the following merging operation according to Zadeh's extension principle [5]. $\pi_{\mathrm{C}}=\pi_{\mathrm{A}} \cup_{f} \pi_{\mathrm{B}}=\left\{\pi_{\mathrm{C}}(\mathrm{w}) / \mathrm{w} \mid\left(\exists \pi_{\mathrm{A}}\left(\mathrm{u}_{\mathrm{i}}\right) / \mathrm{u}_{\mathrm{i}}\right)\left(\exists \pi_{\mathrm{B}}\left(\mathrm{v}_{\mathrm{j}}\right) / \mathrm{v}_{\mathrm{j}}\right)\left(\pi_{\mathrm{C}}(\mathrm{w})=\operatorname{Max}\left(\pi_{\mathrm{A}}\left(\mathrm{u}_{\mathrm{i}}\right)\right.\right.\right.$,

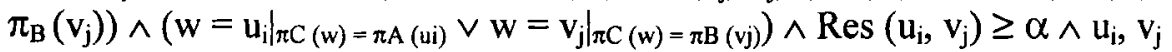
$\in \mathrm{U} \wedge 1 \leq \mathrm{i}, \mathrm{j} \leq \mathrm{n}) \vee\left(\exists \pi_{\mathrm{A}}\left(\mathrm{u}_{\mathrm{i}}\right) / \mathrm{u}_{\mathrm{i}}\right)\left(\forall \pi_{\mathrm{B}}\left(\mathrm{v}_{\mathrm{j}}\right) / \mathrm{v}_{\mathrm{j}}\right)\left(\pi_{\mathrm{C}}(\mathrm{w})=\pi_{\mathrm{A}}\left(\mathrm{u}_{\mathrm{i}}\right) \wedge \mathrm{w}=\mathrm{u}_{\mathrm{i}} \wedge\right.$ $\left.\operatorname{Res}\left(u_{i}, v_{j}\right)<\alpha \wedge u_{i}, v_{j} \in U \wedge 1 \leq i, j \leq n\right) \vee\left(\exists \pi_{B}\left(v_{j}\right) / v_{j}\right)\left(\forall \pi_{A}\left(u_{i}\right) / u_{i}\right)\left(\pi_{C}\right.$ $\left.\left.(w)=\pi_{B}\left(v_{j}\right) \wedge w=v_{j} \wedge \operatorname{Res}\left(u_{i}, v_{j}\right)<\alpha \wedge u_{i}, v_{j} \in U \wedge 1 \leq i, j \leq n\right)\right\}$

Informally, any two tuples in a nested relation are redundant, if, for pair of the corresponding attribute values, the equivalence degree is greater than or equal to the threshold value. If the pair of the corresponding attribute values is simple, the equivalence degree is one for two values. For two values 
of structured attributes, however, the equivalence degree is one for structured attributes. Two redundant tuples $t$ and $t^{\prime}$ are written $t \equiv t^{\prime}$.

\section{RELATIONAL ALGEBRA OPERATIONS}

Union and Difference: Let $r$ and $s$ be two union-compatible fuzzy nested relations. Then

$$
r \cup s=\min (\{t \mid t \in r \vee t \in s\}) \text { and } r-s=\{t \mid t \in r \wedge(\forall \mathrm{v} \in s)(t \neq v)\}
$$

Here, the operation min () means to remove the fuzzy redundant tuples in $r$ and $s$. Of course, the threshold value should be provided for the purpose.

Cartesian Product. Let $r(\mathrm{R})$ and $s(\mathrm{~S})$ be two fuzzy nested relations. Then $r \times s$ is a fuzzy nested relation with the schema $R \cup S$.

$$
r \times s=\{t \mid t(R) \in r \wedge t(S) \in s\}
$$

Projection. Let $r(\mathrm{R})$ be a fuzzy nested relation and $S \subset R$.

$$
\Pi_{S}(r)=\min (\{t \mid(\forall \mathrm{v} \in r)(t=v(S)\})
$$

Here, an attribute in $S$ may be of the form B.C, in which $B$ is a structured attribute and $C$ is its component attribute.

Selection. In classical relational databases, the selection condition is of the form $\mathrm{X} \theta \mathrm{Y}$, where $\mathrm{X}$ is an attribute, $\mathrm{Y}$ is an attribute or a constant value, and $\theta \in\{=, \neq,>, \geq,<, \leq\}$. In order to implement fuzzy query for fuzzy relational databases, " $\theta$ " should be fuzzy, denoting $\approx, \neq, \succ, \prec, \succeq$, and $\preceq$. In addition, $X$ is only a simple attribute or the simple attribute of a structured attribute but $\mathrm{Y}$ may be (a) a constant, crisp or fuzzy one, (b) a simple attribute, or (c) a simple component attribute of a structured attribute, having the form A. B, where $A$ is a structured attribute and $B$ is its simple component attribute.

The fuzzy comparison operations are defined as follows. (a) $\mathrm{X} \approx \mathrm{Y}$ iff $\mathrm{SE}_{\alpha}$ $(X, Y) \geq \beta$, where $\beta$ is a selected cut (the followings are the same); (b) $X \neq Y$ iff $\mathrm{SE}_{\alpha}(\mathrm{X}, \mathrm{Y})<\beta$; (c) $\mathrm{X} \succ \mathrm{Y}$ iff $\mathrm{X} \neq \mathrm{Y}$ and $\min (\operatorname{Supp}(\mathrm{X}))>\min (\mathrm{Supp}$ (Y)); (d) $X \succeq Y$ iff $X \approx Y$ or $X \succ Y$; (e) $X \prec Y$ iff $X \neq Y$ and $\min (\operatorname{Supp}(X))$ $<\min (\operatorname{Supp}(\mathrm{Y})) ;(\mathrm{f}) \mathrm{X} \preceq \mathrm{Y}$ iff $\mathrm{X} \approx \mathrm{Y}$ or $\mathrm{X} \prec \mathrm{Y}$.

Depending on $Y$, the following situations can be identified for $X \theta Y$. Let $X$ be the attribute $A_{i}: \tau_{i}$ in a fuzzy nested relation.

(1) $A_{i} \theta c$, where $c$ is a crisp constant. According to $\tau_{i}, A_{i} \theta c$ is as follows: if $\tau_{\mathrm{i}}$ is dom, then $\theta \in\{=, \neq,>,<, \geq, \leq\}$; if $\tau_{\mathrm{i}}$ is fdom, then $\theta \in\{\approx, \neq, \succ, \prec$, $\succeq, \preceq\}$; if $\tau_{\mathrm{i}}$ is ndom, $\mathrm{A}_{\mathrm{i}} \theta c$ is a null comparison and regarded as the special fuzzy comparison; if $\tau_{i}$ is sdom, $A_{i} \theta c$ is a element-set comparison. Then $A_{i} \theta$ $c$ if $c$ and any element in the value of Ai of a tuple satisfy the " $\theta$ ".

(2) $\mathrm{A}_{\mathrm{i}} \theta f$, where $f$ is a fuzzy value: if $\tau_{\mathrm{i}}$ is $d o m$, fdom, or $n d o m, \mathrm{~A}_{\mathrm{i}} \theta f$ is a fuzzy comparison and $\theta \in\{\approx, \neq, \succ, \prec, \succeq, \preceq\}$; if $\tau_{\mathrm{i}}$ is sdom, $\mathrm{A}_{\mathrm{i}} \theta f$ is a fuzzy set comparison. Then $\mathrm{A}_{\mathrm{i}} \theta f$ if $f$ and any element in the value of $\mathrm{Ai}$ of a tuple satisfy the fuzzy " $\theta$ ", where $\theta \in\{\approx, \neq, \succ, \prec, \succeq, \preceq\}$. 
(3) $A_{i} \theta A_{j}$, where $A_{j}: \tau_{j}$ is a simple attribute and $i \neq j$ : if $\tau_{i}$ and $\tau_{j}$ are all dom, $\mathrm{A}_{\mathrm{i}} \theta \mathrm{A}_{\mathrm{j}}$ is a traditional comparison; if $\tau_{\mathrm{i}}$ and $\tau_{\mathrm{j}}$ are dom and fdom, fdom and fdom, or ndom and fdom, $\mathrm{A}_{\mathrm{i}} \theta \mathrm{A}_{\mathrm{j}}$ is a fuzzy comparison; if $\tau_{\mathrm{i}}$ and $\tau_{\mathrm{j}}$ are dom and ndom, $\mathrm{A}_{\mathrm{i}} \theta \mathrm{A}_{\mathrm{j}}$ is a null comparison; if $\tau_{\mathrm{i}}$ and $\tau_{\mathrm{j}}$ are dom and sdom, $\mathrm{A}_{\mathrm{i}}$ $\theta \mathrm{A}_{\mathrm{j}}$ is a element-set comparison; if $\tau_{\mathrm{i}}$ and $\tau_{\mathrm{j}}$ are fdom and sdom, $\mathrm{A}_{\mathrm{i}} \boldsymbol{\theta} \mathrm{A}_{\mathrm{j}}$ is a fuzzy set comparison; if $\tau_{i}$ and $\tau_{j}$ are all ndom, $A_{i} \theta A_{j}$ is a null-null comparison. Then $A_{i} \theta A_{j}$ if they have the same null values on the same universe of discourse; if $\tau_{i}$ and $\tau_{j}$ are ndom and sdom, $A_{i} \theta A_{j}$ is a null-set comparison and regarded as the special element-set comparison; if $\tau_{i}$ and $\tau_{j}$ are sdom and sdom, $\mathrm{A}_{\mathrm{i}} \theta \mathrm{A}_{\mathrm{j}}$ is a set-set comparison and regarded as the special element-set comparison.

(4) $A_{i} \theta A_{j}$. B, where $A_{j}$ is a structured attribute $(i \neq j)$ and $B$ is a simple attribute. The situations are the same as those in above case (3).

Let $Q$ be a predicate denoting the selection condition. The selection operation for a fuzzy nested relation $r$ is defined as $\sigma_{\mathrm{Q}}(r)=\{t \mid t \in r \wedge Q(t)\}$

We do not give the definitions of two restructuring Nest and Unnest in the database model because of the space limitation.

\section{CONCLUSION}

In this paper, we introduced fuzzy data into nested relational databases to model complex objects and imprecise and uncertain information. The fuzzy data here is extended possibility-based fuzzy data expressed by possibility distribution over a universe of discourse and meanwhile a resemblance relation on the universe is in effect. We gave the structure of fuzzy nested relational model and the approach for assessing fuzzy data redundancies. On the basis, the algebra is defined for manipulating fuzzy and complex data.

\section{REFERENCES}

[1] M. Levene, The Nested Universal Relation Database Model, Lecture Notes in Computer Science No. 595, Springer-Verlag, Berlin, 1992.

[2] Z. M. Ma, W. J. Zhang and W. Y. Ma, Semantic Measure of Fuzzy Data in Extended Possibility-Based Fuzzy Relational Databases, International Journal of Intelligent Systems, 15 (8) (2000) 705-716.

[3] A. Yazici, A. Soysal, B. P. Buckles and F. E. Petry, Uncertainty in a Nested Relational Database Model, Data \& Knowledge Engineering, 30 (1999) 275-301.

[4] L. A. Zadeh, Fuzzy Sets, Information and Control, 8 (3) (1965) 338-353.

[5] L. A. Zadeh, The Concept of a Linguistic Variable and Its Application to Approximate Reasoning (Parts 1, 2, and 3), Information Science, 8 (1975) 119-248, 301-357; 9 (1975) 43-80. 